\title{
A Boundary Element Study for Evaluation of the Effects of the Rigid Baffles on Liquid Sloshing in Rigid Containers
}

\author{
Amir Behshad*1, Mohammad Reza Shekari ${ }^{2}$ \\ ${ }^{I}$ Faculty of Industry and Mining, Yasouj University, Choram, Iran, a.behshad@mail.yu.ac.ir \\ ${ }^{2}$ Department of Civil Engineering, Estahban Higher Education Center, Estahban, Iran, shekari.2291@gmail.com
}

\section{ARTICLE INFO}

Article History:

Received: 5 Oct. 2017

Accepted: 10 Sep. 2018

Keywords: Liquid sloshing; baffled tank; boundary element method

\begin{abstract}
In this paper, the sloshing response of liquid in a two dimensional rigid rectangular tank with rigid baffles is investigated using boundary element technique. A baffle is a supplementary structural element which supplies a kind of passive control on the effects of ground shaking. The complicated liquid domain is divided into two simple sub-domains so that the liquid velocity potential in each liquid sub-domain is specified employing Green's theorem, and the walls and free surface boundary conditions are applied. The liquid region is modeled by internal quadrilateral boundary elements, which reduce the three-dimensional fluid problem into a two-dimensional-surface one. The validity of the present algorithm is assessed through the comparison with the accessible results for the rectangular tank without baffle and then developed to the solution of tanks with rigid baffles. Several parametric studies are performed to show the liquid sloshing effects in terms of the slosh frequencies and free surface displacement by consideration of the effects of baffle parameters such as position and dimension. From these analyses, it may be concluded that in the special case of long-period ground earthquake, the baffle device amplifies the dynamic responses of liquid tank which may be interpreted by the fact that the predominant period of the ground shaking is set at the fundamental natural sloshing periods.
\end{abstract}

\section{Introduction}

Partially filled liquid containers undergoing ground excitations are known to exhibit the phenomenon of sloshing. Accelerated motions of the contained liquids can result in substantial slosh induced loads, which may adversely affect the dynamic behavior of the liquid-retaining structures, principally, in the vicinity of resonances [1]. Thus, it is still urged the need for further research on the cognizing of the physical behavior of liquid sloshing and the factors affecting the sloshing behavior of liquids is of considerable importance in the process of design of such tanks. Generally the amplitude of the slosh depends on several parameters such as liquid properties and tank geometry, liquid depth, and amplitude and frequency of the tank motion, which have direct effects on the dynamic behavior of moving tanks. Evans and McIver [2] examined the effect of introducing a vertical baffle into a rectangular container on fluid frequencies by employing the appropriate eigenfunction expansions and afterwards, Watson and Evans [3] developed this technique for a number of similar problems. Choun and Yun [4,5] investigated the effects of the size and position of a submerged block on the sloshing characteristics of the fluid in a rectangular tank using the linear water wave theory. Their results showed that the wave surface displacement increases in the district of the block, the sloshing frequencies generally decrease as the block becomes tall and wide, and a large hydrodynamic pressure acts on the tank wall and block when the block moves toward the wall. Warnitchai and Pinkeaw [6] developed a mathematical model of liquid sloshing confirmed by experimental investigations in rectangular tanks, which includes the effects of flow-dampening devices and concluded that a high damping of sloshing can happen using the vertical flat plate and the wire mesh screen. Isaacson and Premasiri [7] developed the mathematic solutions accompanied with experiment investigations to describe the hydrodynamic damping due to baffles in a fluid-filled rectangular tank subjected to horizontal oscillations. The average rate of energy dissipation due to flow separation around baffles and the total energy of sloshing waves are employed to assess the hydrodynamic damping. 
Employing finite element method, Biswal et al. [8] investigated the liquid sloshing in a rigid rectangular tank with and without baffles under sinusoidal base excitation, and afterwards in 2004 expanded a finite element formulation to compute the slosh amplitude of liquid in a partially liquid-filled cylindrical tank with and without a rigid baffle undergoing translational base excitation [9].

Several studies related to liquid sloshing have been carried out numerically along with the development of microprocessors and computational techniques in the recent decades. The usage of such procedures as the finite difference, finite volume, finite element, boundary element and pseudo-spectral methods has become very customary in this field. Frandsen [10] developed a finite difference model based on inviscid flow equations accompanied with numerical experiments of sloshing wave motion in a 2-D tank which is excited both horizontally and vertically. Bermudez et al. [11] proposed a finite element algorithm to approximate the vibration modes of a plate in contact with an incompressible fluid in which, the effect of the fluid is simplified by added masses. Lee and Cho [12] studied on the structural dynamic response of moving fuel-storage tanks with baffles employing (ALE) finite element method that is extensively being used to handle the problems with free surface, moving boundary, large deformation, and interface contact. Among numerical procedures, the boundary element method (BEM) has worked out to be well popular and has had prosperous applications in the simulations of liquid sloshing problems [13-15]. Discretizing the boundaries instead of discretizing the entire liquid region and subsequently cutting down on the costs of memory and time is one of the most important reasons for favoring the application of BEM over other methods.

Gedikli and Erguven [16,17] investigated the effects of a rigid baffle on the natural modes of liquid in a rigid cylindrical tank using direct BEM and method of superposition of modes. Kita et al. [18] described the application of Trefftz-type BEM for the simulation of the sloshing phenomenon of the fluid in a rectangular vessel. Assuming a laminar boundary layer, Jamali [19] presented a solution to the liquid surface motion through a combinatory use of BEM and the finite difference method and then calculated the damping rate. Ning et al. [20] simulated wave tank using the boundary integral equation method (BIEM) and computed the solid angle coefficients. Employing boundary element method, Sygulski [21] studied the natural frequencies and mode shapes of liquid sloshing in three dimensional baffled tanks with arbitrary geometries. Ebrahimian et al. [22] developed a Boundary Element algorithm to determine the symmetric and antisymmetric sloshing natural frequencies and mode shapes for multi baffled axisymmetric tanks with arbitrary geometries. Some researches related to liquid oscillations have been carried out numerically, in the past several decades [23, 24].

The above literature review evidently indicates that there exists a very limited literature on sensitivity of baffled tank seismic responses to long period earthquake records with different frequency contents. Thus, the present study tries to fulfill this mission to an acceptable extent.

As an extension of the previous researches, the effects of rigid baffles on the sloshing of an inviscid, incompressible liquid in rectangular tanks are numerically studied by boundary element method subjected to horizontal base excitations. The amplitudes of oscillations are supposed to be small. Having followed the technique that is illustrated in this paper, a numerical model is attained that is represented only in terms of the potential values of liquid free surface nodes, which is computationally more efficient than a full boundary element pattern. The introduced technique is verified in comparison with the literature and used to carry out an extensive examination of the effect of baffle on sloshing responses of rectangular liquid storage tanks.

\section{Governing equations}

The dynamic of fluid is explained completely by Navier-Stokes equations and the non-slipping condition on the walls and kinematic and dynamic conditions of the free surface as boundary conditions. Although these equations depict a complete sketch of the fluid and phenomena related to it, but solving them have been always an important concern for researchers worked on fluid dynamics filed and no general analytical solution has been proposed for them. There are some further applicable patterns to inspect the behavior of the fluid such as potential flow which is stated for irrotational flow. Based on existence of a function as potential of velocity and using Navier-Stokes equations for inviscid and irrotational flow, the governing equation of sloshing of an incompressible fluid in a three dimensional tank is expressed by the Laplace equation $[25,26]$ :

$\nabla^{2} \varphi=0$

In which, $\varphi$ is the velocity potential function. Assuming the tank wall to be rigid, the boundary condition at the fluid-structure interface is described as follows:

$$
\frac{\partial \varphi}{\partial n}=0
$$

Linearized kinematic and dynamic boundary conditions of the free surface of the contained liquid are considered as follows:

$$
\frac{\partial \varphi}{\partial z}=\frac{\partial \eta}{\partial t}
$$


(a)

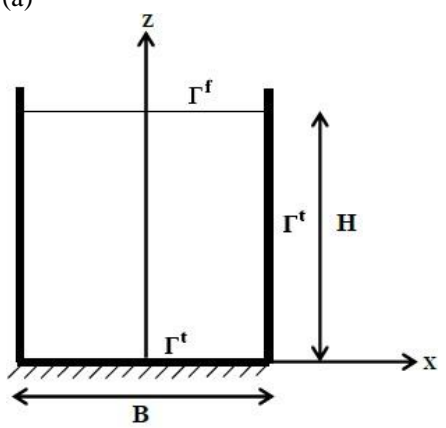

(b)

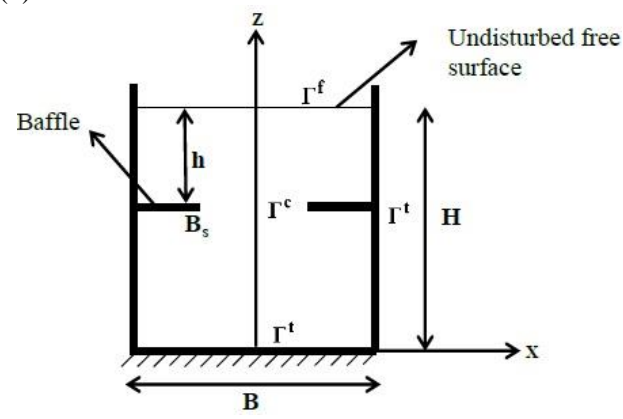

Fig. 1. Cross-section of rectangular container. a) Without baffle, b) with baffle.

$$
\frac{\partial \varphi}{\partial t^{2}}+g \eta=0
$$

where $n$ is the normal of the surface pointing out of the liquid domain, $\mathrm{z}$ denotes vertical Cartesian coordinate axis, $\eta$ is the sloshing height at liquid free surface $(\mathrm{z}=0), \mathrm{g}$ indicates the gravitational acceleration, and $t$ represents the time.

The hydrodynamic pressure acting on the tank wall may be acquired using the linearized Bernoulli equation as

$P=-\rho \frac{\partial \varphi}{\partial t}$

Moreover, the hydrodynamic pressure at the liquid free surface is calculated by

where $\rho$ the mass density of the liquid.

\section{Developing the BEM formulation for baffled rectangular tanks}

The formulation specified above has no restriction on the shape of the container, i.e. it may have any shape. A rectangular container with/without a baffle is taken into consideration as an application to the presented formulation in the rest of this study, (Fig. 1).

By employment Green's equation and divergence theorem and assuming that $\mathrm{f}$ and $q$ are well-behaved functions; the following integral equation is attainable [24]:

$\alpha_{\mathrm{P}} \phi(P)+\int_{\Gamma}\left(\phi \frac{\partial G^{*}}{\partial n}-G^{*} \frac{\partial \phi}{\partial n}\right) d \Gamma=0$

Here $G^{*}, \partial G^{*} / \partial n$ are the fundamental solution of the Laplace equation for the axially-asymmetric problem and its derivative, respectively, and $\alpha_{\mathrm{P}}$ is defined by the position of the source point $P$. By appraising the integrals in equation (7) over boundary elements, the following linear system of equations is achieved [27]:

$H_{i k}=\int_{\Gamma} G_{i, n}^{*}(P, s) r(s) d s$

$G_{i k}=\int_{\Gamma} G_{i}^{*}(P, s) r(s) d s$

where $G_{i}^{*}$ is called the free space Green function which is the potential on the point $S$ due to the unit source placed on point P. Discretized form of boundary element equations of the liquid region may be written as the following expressions, according to the free surface and tank nodes of the liquid region [23]:

$$
\left[\begin{array}{ll}
\mathbf{H}_{t t} & \mathbf{H}_{t f} \\
\mathbf{H}_{f t} & \mathbf{H}_{f f}
\end{array}\right]\left[\begin{array}{l}
\phi_{t} \\
\phi_{f}
\end{array}\right]=\left[\begin{array}{ll}
\mathbf{G}_{t t} & \mathbf{G}_{t f} \\
\mathbf{G}_{f t} & \mathbf{G}_{f f}
\end{array}\right]\left[\begin{array}{l}
\mathbf{q}_{t} \\
\mathbf{q}_{f}
\end{array}\right]
$$

where the subscripts $t$ and $f$ show the boundary nodes on the tank wall and liquid free surface, respectively (see Fig. 1).

\section{Illustrative numerical results}

A boundary element code is developed to evaluate the sloshing response and hydrodynamic pressure in the partially liquid filled containers with and without baffles. Several liquid storage tanks with various properties are inspected to demonstrate the validity of the proposed modeling process. After validation, the present test program is used to compute the liquid sloshing responses in the tanks with rigid baffles.

\subsection{BEM code verification}

We first accomplish the verification experiments with liquid-filled tanks without baffle, in order to validate the boundary element method introduced and the test program developed. The following analytical expression gives the sloshing frequencies of a vertical cubic liquid tank [1]:

$\omega_{m n}^{2}=\frac{g \pi}{a} \sqrt{m^{2}+n^{2}} \tanh \frac{\pi H \sqrt{m^{2}+n^{2}}}{a}$

Fig. 2 plots the variation of the lowest four sloshing frequencies against liquid depth and gives a comparison between the present BEM and the exact results. The results of the present study are in reasonable agreement with the results of Ibrahim [1]. 


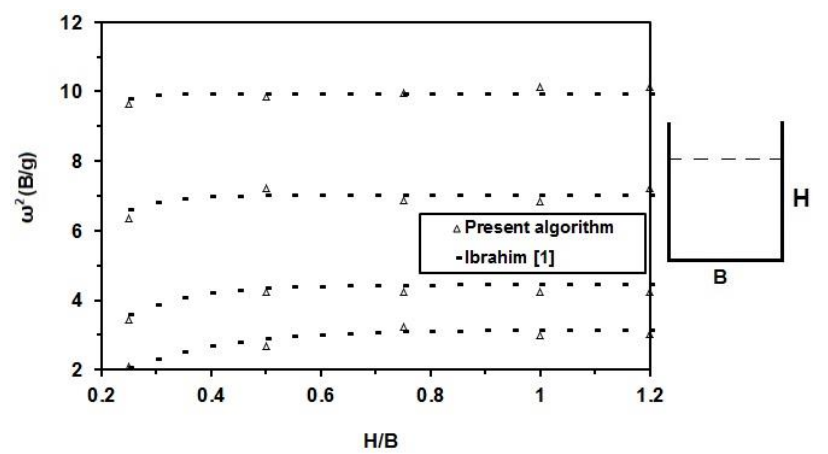

Fig. 2. Dimensionless sloshing frequencies for various liquid depths.

A baffled rectangular container is the second surveyed case which its results is validated with those of Biswal et al. [28]. The liquid height and the tank width are set by $\mathrm{H}=0.5 \mathrm{~m}$ and $\mathrm{B}=1 \mathrm{~m}$, respectively. The tank is subjected to sinusoidal horizontal excitation, $\ddot{u}_{g}(t)=\ddot{u}_{g o} \omega^{2} \sin (\omega t) \quad$ where $\quad \ddot{u}_{g o}=0.002 m \quad$ and $\omega=5.29 \mathrm{rad} / \mathrm{s}$. A single baffle with thickness of $0.005 \mathrm{~m}$ is placed is placed at a depth of $h$ from the liquid free surface. Fig. 3 shows the time variation of free surface sloshing response at the right wall of the tank for $h / H=0.1$. It is observed from the Fig. 3 that the present BEM results compare well with the FEM results of Biswal et al. [28] and a close agreement of results has been perceived.

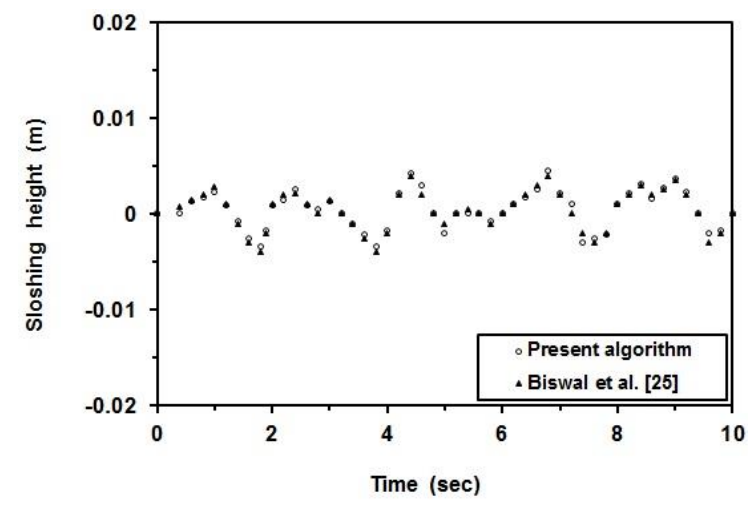

Fig. 3. Liquid sloshing height at tank wall in a rectangular tank under sinusoidal base excitation.

The accurateness of the introduced numerical strategy is examined in the third case for a seismic excitation, using the data of Goudarzi and Sabbagh-Yazdi [29].

The results of this numerical algorithm for liquid surface wave are compared with the results of the analogous experimental data. These comparisons for the free surface oscillation at the left wall of the tank are exposed in Fig. 4.

Indeed, the experiments were performed with water depth equal to $0.624 \mathrm{~m}$ and aspect ratio (H/a) of 0.4 , respectively. Ultrasonic wave gauges were employed to detect the wave elevations on the both side walls of the tank and the displacement of the shaking table. The sloshing amplitude of the rectangular tank was studied under the harmonic sinusoidal vibration with the relative frequency of 1.07 . In order to quantitatively evaluate the preference of the present algorithm against benchmark experimental data, the square of the correlation factor $\left(\mathrm{R}^{2}\right)$ is employed for which a squared correlation factor close to 1 denotes full agreement. In fact, the square of the correlation factor between measured and computed sloshing height is about $93.8 \%$. As can be perceived, the numerical results are in good agreement with the experimental measurements that approves the validity of the numerical procedure.

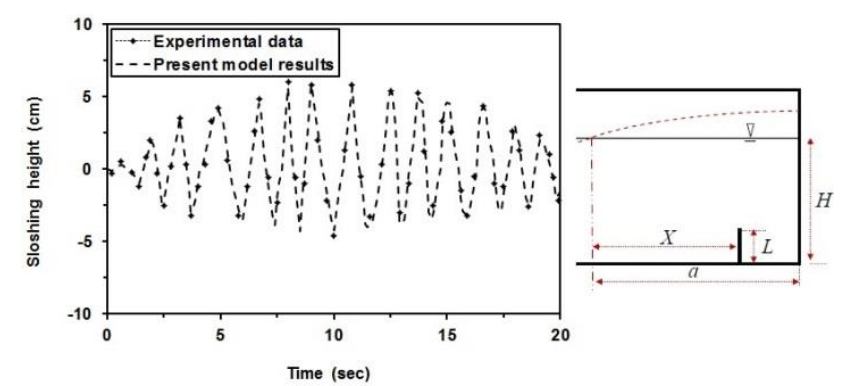

Fig. 4. Comparison of the wave height with the experimental results of Goudarzi and Sabbagh-Yazdi [29].

The results of the present work for liquid pressure variation are compared with the results of the analogous experimental data, for unbaffled rectangular tank. Here, the water depth is taken as $0.31 \mathrm{~m}$, the tank width $0.46 \mathrm{~m}$, and the tank length $0.92 \mathrm{~m}$. The pressure amplitude at the bottom of the tank was assessed under the harmonic sinusoidal vibration with the frequency of $2 \mathrm{rad} / \mathrm{s}$. In Figs. 5, it can be concluded that the present time simulation of the pressures at bottom of the tank give a rational agreement with the experimental results of Akyildiz and Unal [31].

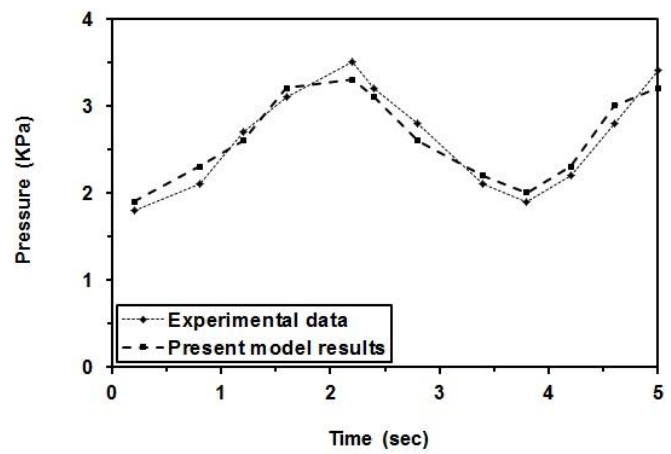

Fig. 5. Comparison of the pressure with the experimental results of Akyildiz and Unal [31].

\subsection{Parametric experiments}

When a container is subjected to linear instability, a series of surface waves are arisen in the liquid and thus the liquid exerts a pressure on the walls of the container. In the present investigation, the effect of horizontal baffles on the level of displacement of the free surface and sloshing natural frequencies has been studied. 


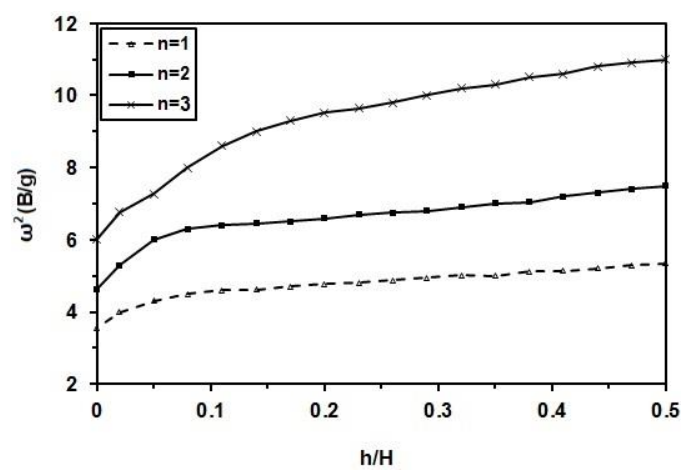

a) $B_{\mathrm{s}} / B=0.1$

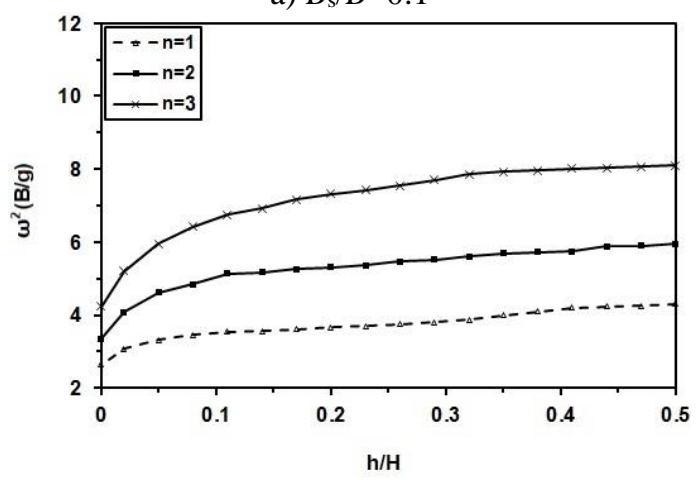

c) $B_{\mathrm{s}} / B=0.3$

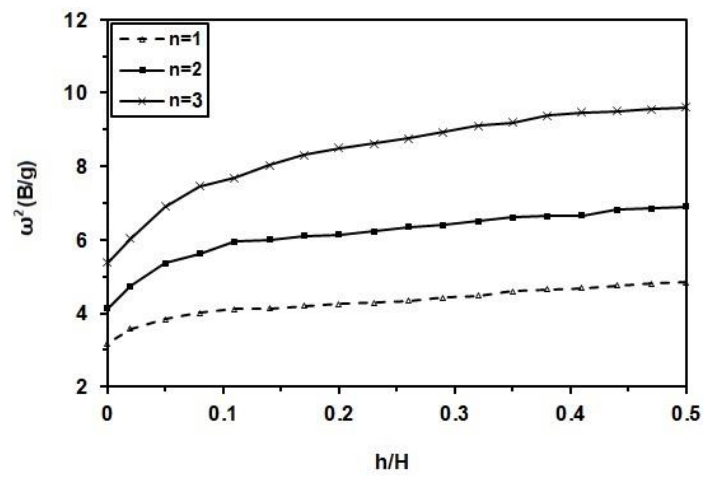

b) $B_{\mathrm{s}} / B=0.2$

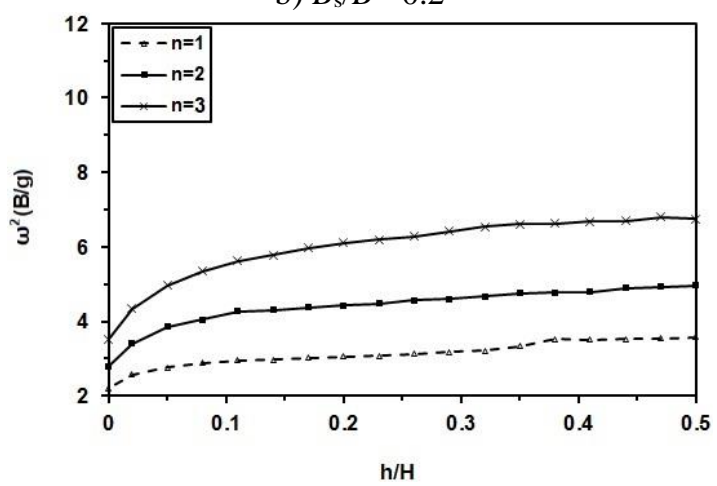

d) $B_{\mathrm{s}} / B=0.4$

Fig. 6. The natural frequencies of the liquid in the rectangular container vs. the baffle position $(H / B=1.0)$.

\subsubsection{Sloshing frequency variation with different baffle orientations}

The natural frequencies of a cubic tank corresponding to the first three modes are plotted for the various baffle widths and positions, Fig. 6. The minimum dimensionless sloshing frequencies, assuming the first mode, are 3.56, 3.17, 2.65 and 2.21 when the relative baffle widths $\left(B_{s} / B\right)$ are $0.1,0.2,0.3$ and 0.4 , respectively. For the second mode, for the foregoing baffle widths, the corresponding sloshing frequencies are $4.62,4.13,3.35$ and 2.79 , respectively. The analogous sloshing frequencies, assuming the third mode, are $6.01,5.37,4.23$ and 3.52 , respectively. Results expose a decrease in frequencies as the baffle nears the free surface, which can be elucidated as the effect of the shallow liquid region that is formed on the baffle. Moreover, the comparative results indicate that the sloshing frequencies decrease considerably as the relative baffle width increases.

\subsubsection{Effects of horizontal excitation on sloshing response}

In this section, liquid sloshing subjected to horizontal excitation, with and without a horizontal baffle, will be methodically inspected. The width and liquid depth in the tank are taken as $B=1 \mathrm{~m}$ and $H=0.7 \mathrm{~m}$, respectively. The horizontal excitation is taken as $x_{b}=x_{0} \cos \left(\omega_{h} t\right)$, where $x_{0}$ and $\omega$ are the amplitude and frequency of the forced displacement, respectively. The horizontal excitation amplitude is taken as 0.003 and $0.03 \mathrm{~m}$ corresponding to small and large excitation amplitude and the parameter $\omega$ as 5.48 $\mathrm{rad} / \mathrm{s}$. The liquid surface displacement variations at the right wall of the tank are computed for various excitation amplitudes, $B_{\mathrm{s}} / B=0.4$ and $h / H$ ratios equal to $0.15,0.4,0.65$ and 0.9 as illustrated in Figs. 7 and 8. The peak sloshing heights, assuming excitation amplitude of $0.003 \mathrm{~m}$, are $1.62,2.16,2.71$ and $6.81 \mathrm{~cm}$ when the $h / H$ ratios are $0.15,0.4,0.65$ and 0.9 , respectively. Similarly, for the excitation amplitude of $0.03 \mathrm{~m}$, the corresponding peaks are $2.34,3.78,4.73$ and $7.95 \mathrm{~cm}$, respectively. The above results indicate that the sloshing height at the tank wall increases with the increase in the $h / H$ ratios. This could be justified by the fact that the baffle is moved away from the free surface as the $h / H$ ratio increases therefore the moving fluid has enough kinetic energy to climb up. Also the results indicate that the peak sloshing displacement show relatively considerable increase when the $h / H$ ratio is 0.9 , which may be attributed to the fact that the first sloshing mode frequency (i.e., $\omega_{1}=5.45 \mathrm{rad} / \mathrm{s}$ ) is close to the frequency of the forced excitation (i.e., $5.48 \mathrm{rad} / \mathrm{s}$ ). 


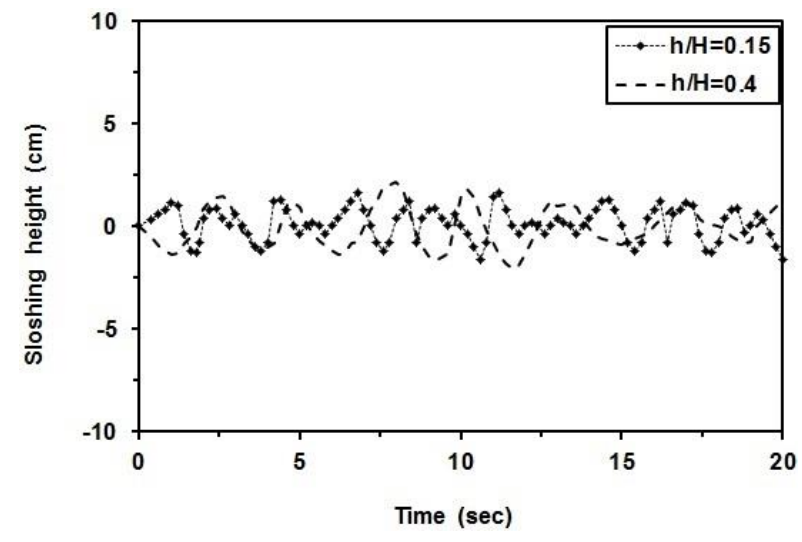

a) $h / H=0.15$ and 0.4

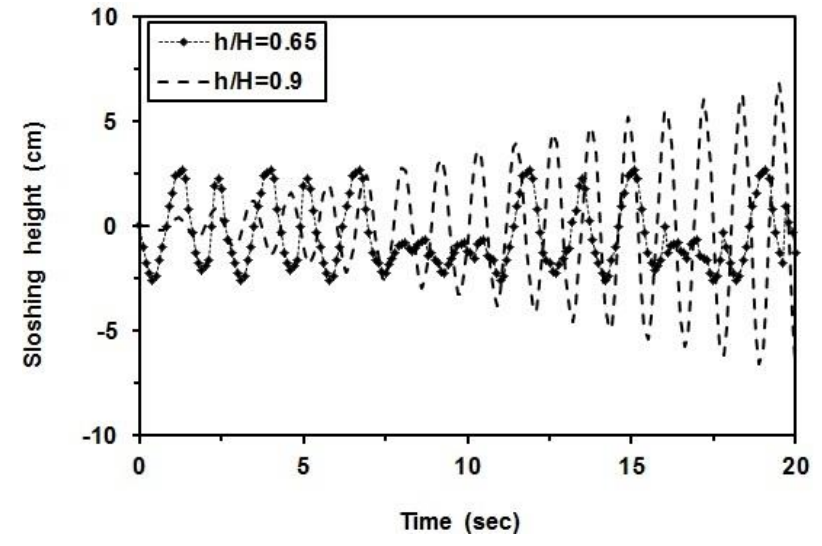

b) $h / H=0.65$ and 0.9

Fig. 7. Liquid sloshing height time histories for $x_{0}=0.003 \mathrm{~m}$ and various baffle positions.

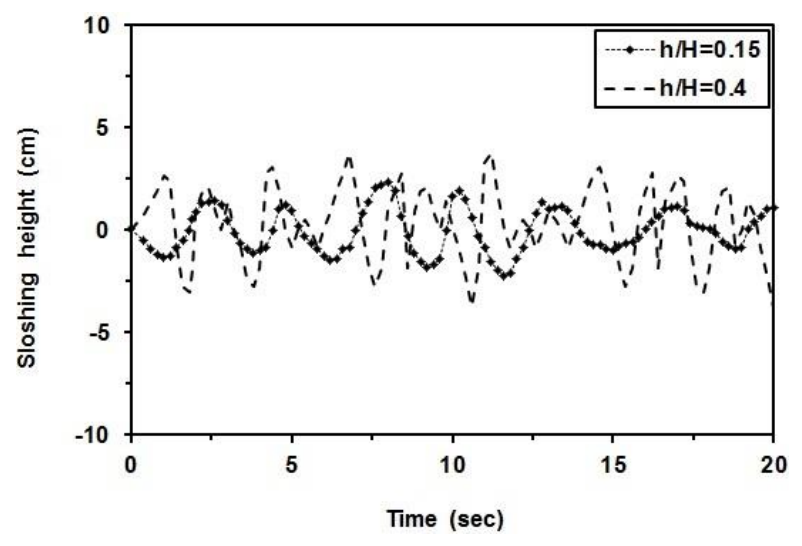

a) $h / H=0.15$ and 0.4

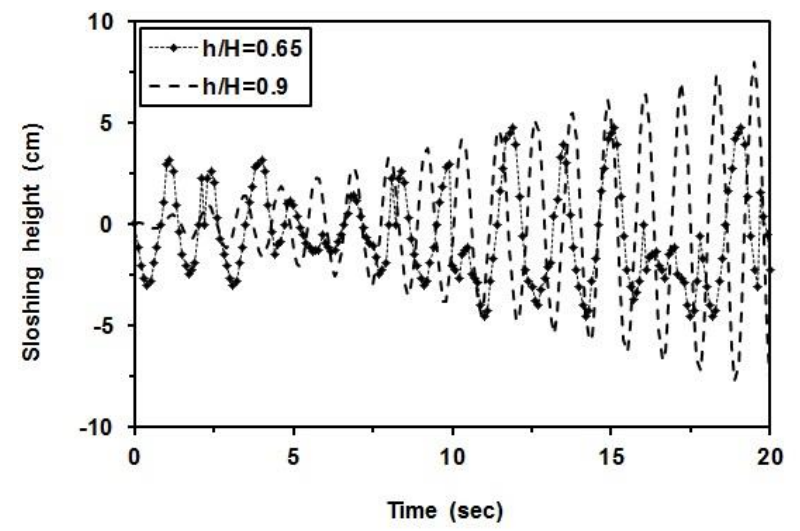

b) $h / H=0.65$ and 0.9

Fig. 8. Liquid sloshing height time histories for $x_{0}=0.03 \mathrm{~m}$ and various baffle positions.

The parametric studies are carried out by varying the width of baffle in the tank wall. The liquid sloshing heights at the right wall of the tank are computed for the excitation amplitude of $0.03 \mathrm{~m}$, excitation frequency of $5.48 \mathrm{rad} / \mathrm{s}, h / H=0.4$ and $B_{\mathrm{s}} / B$ ratios equal to $0.1,0.2,0.3$ and 0.4 as presented in Fig. 9.

The maximum sloshing heights are 21.06, 17.81, 13.61 and $11.34 \mathrm{~cm}$ when the $B_{\mathrm{s}} / B$ ratios are $0.1,0.2$, 0.3 and 0.4 , respectively. Comparative results indicate that slosh amplitude at the tank wall decreases with the increase in $B_{\mathrm{s}} / B$ ratios, which could be because of the fact the baffle surface area is increased with the increase in $B_{\mathrm{s}} / B$ ratios.

\subsubsection{Effects of Horizontal and Vertical Excitation on Liquid Sloshing}

As the excitation power is directly related to the sloshing response, the effects of coupled horizontal and vertical excitation is examined in this section.

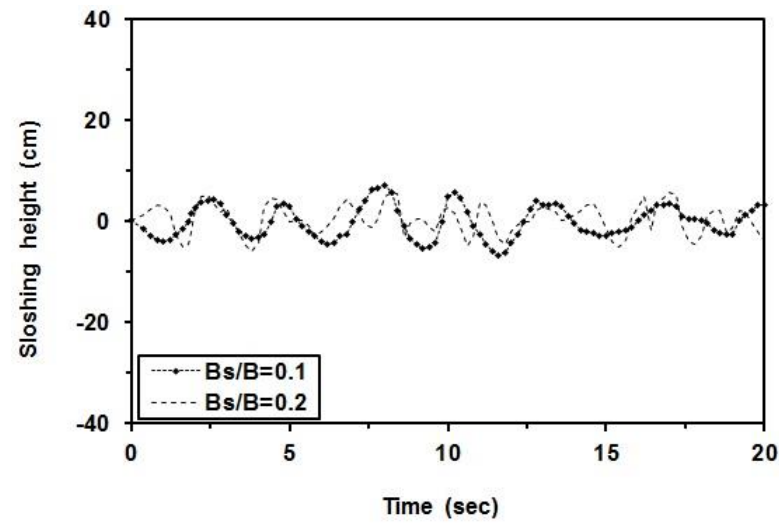

a) $B_{\mathrm{S}} / B=0.1$ and 0.2

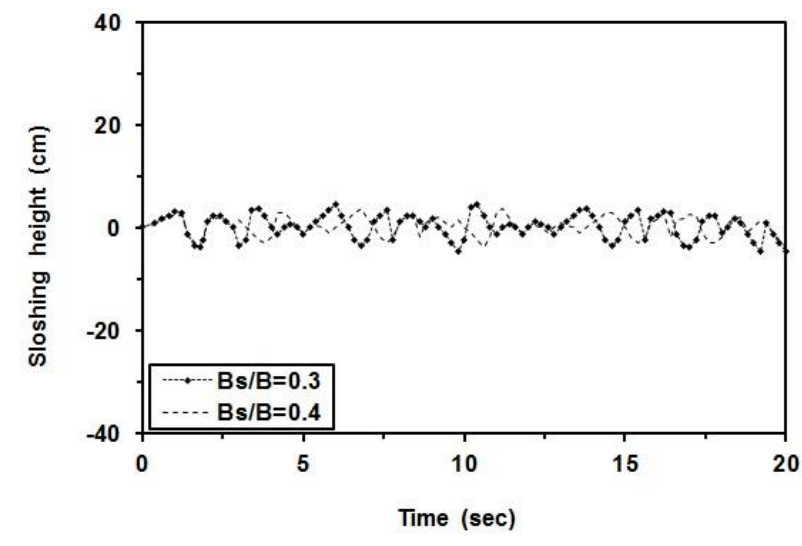

b) $B_{\mathrm{s}} / B=0.3$ and 0.4

Fig. 9. Liquid sloshing time variations for various baffle widths. 


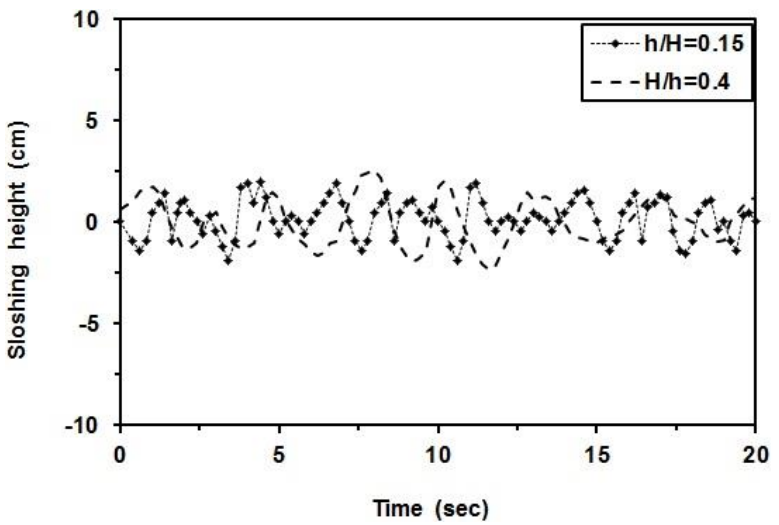

a) $h / H=0.15$ and 0.4

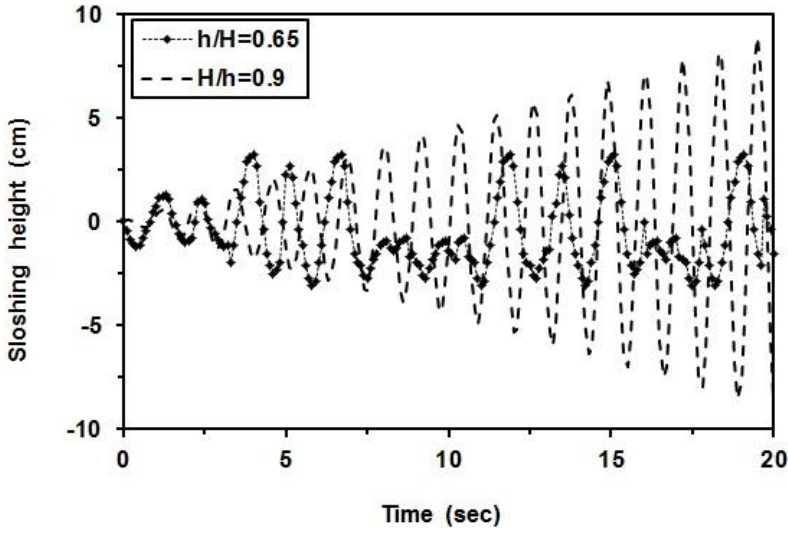

b) $h / H=0.65$ and 0.9

Fig. 10. Liquid sloshing height time variations for $x_{0}=\mathbf{0 . 0 0 3 m}$ and various baffle positions.

Actually, the tank shaking is complicated and can be considered coupled movement of some basic movements. Horizontal and vertical excitations are taken as $x_{b h}=x_{0 h} \cos \left(\omega_{h} t\right) \quad$ and $x_{b v}=x_{0 v} \cos \left(\omega_{v} t\right)$, respectively. The tank dimensions are taken the same as the above case and, an example of $x_{0 \mathrm{~h}}=x_{0 \mathrm{v}}=0.003 \mathrm{~m}$ is considered in this section. Fig. 8 reveals the sloshing height time histories at the right wall of the tank subjected to the total horizontal and vertical excitations. The peak sloshing heights are 1.92, 2.59, 3.24 and $8.86 \mathrm{~cm}$ when the $h / H$ ratios are $0.15,0.4$, 0.65 and 0.9 , respectively. It is noticeable that the closer to the free surface the vertical baffle is, the smaller the total sloshing height is, which coincides with the conclusion of Fig. 8.

Fig. 11 reveals the sloshing height time variations at the right wall of the tank subjected to the vertical excitation. It is obvious that the total vertical sloshing height approximately remains unchanged for different positions of the baffle.

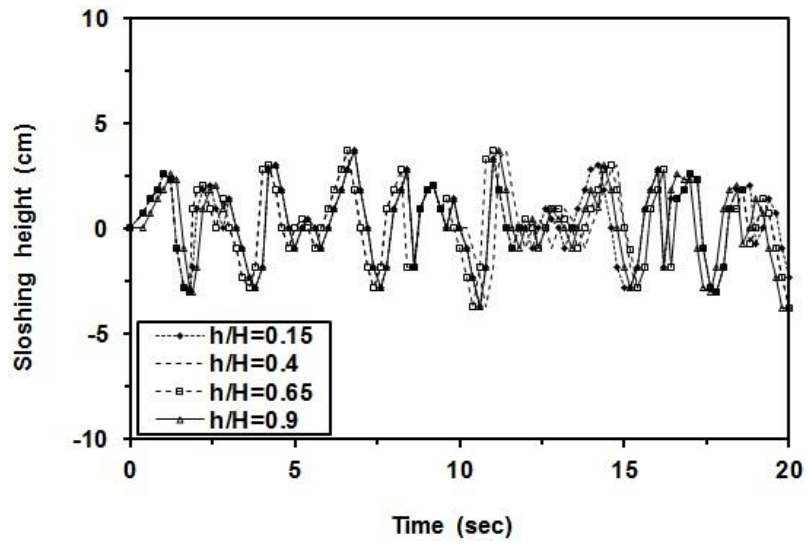

Fig. 11. Liquid sloshing time variations for various baffle positions $\left(B_{\mathrm{s}} / B=0.4\right)$.
4.2.4. Seismic analysis of baffled rectangular tank In this section, the effects of horizontal baffles on the sloshing responses of rectangular liquid storage tanks subjected to the 1995 Kobe earthquake in Japan as a destructive earthquake with a PGA of $0.234 \mathrm{~g}$ are investigated (see Fig. 12). Dynamic analyses are performed to observe the seismic response of a rectangular tank of the same width $B=1 \mathrm{~m}$ and liquid depth $H=0.7 \mathrm{~m}$, with different baffle widths and positions.

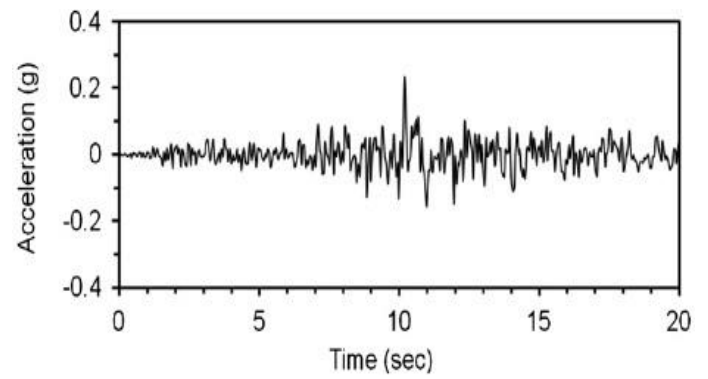

Fig. 12. Ground acceleration time history (1995 Kobe earthquake).

The time variation of liquid surface displacements under foregoing ground motion for various relative baffle heights $(h / H)$ is illustrated in Fig. 13, considering $B_{\mathrm{s}} / B=0.4$. According to the figure, the peak sloshing heights are 4.68, 6.93, 9.01 and 11.72 $\mathrm{cm}$ when the $h / H$ ratios are $0.15,0.4,0.65$ and 0.9 , respectively. One can conclude that the sloshing height at the tank wall is significantly reduced as the $h / H$ ratio decreases so that, at the $h / H$ ratio of 0.15 the reduction is by $60 \%$.

Figs. 14 represent time histories of the sloshing height for various relative baffle widths $\left(B_{\mathrm{s}} / B\right)$, considering $h / H=0.4$. 


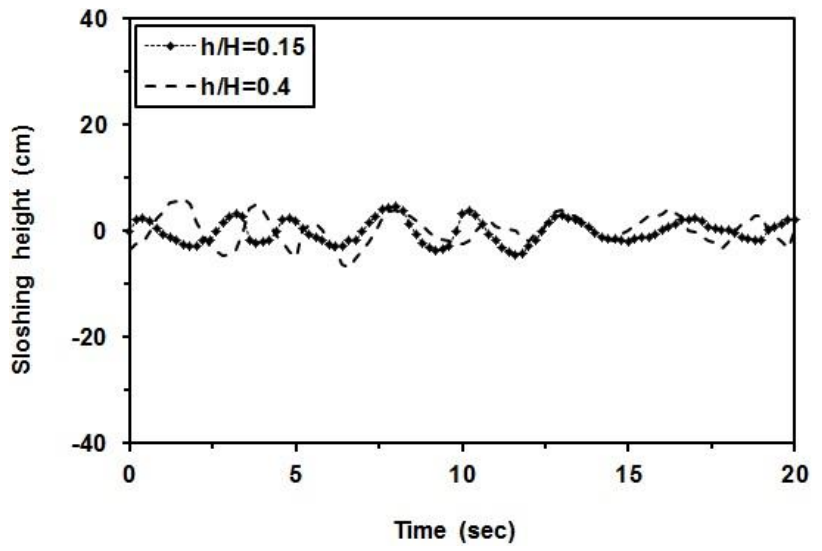

a) $h / H=0.15$ and 0.4

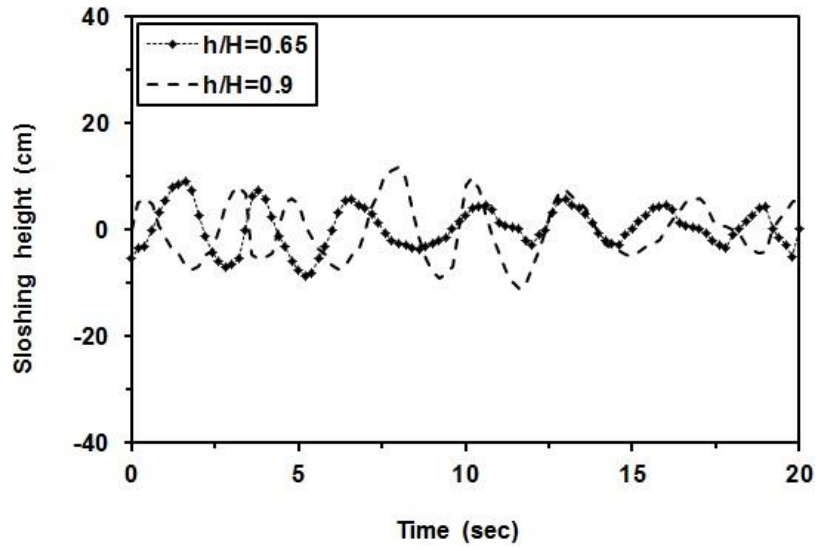

b) $h / H=0.65$ and 0.9

Fig. 13. Liquid sloshing height time histories for various baffle positions under the 1995 Kobe earthquake.

The maximum sloshing heights are $12.63,10.09,8.16$ and $6.93 \mathrm{~cm}$ when the $B_{\mathrm{s}} / B$ ratios are $0.1,0.2,0.3$ and 0.4 , respectively. Comparative results indicate that the sloshing height at the tank wall is significantly reduced as the $B_{\mathrm{s}} / B$ ratio increases so that, at the $B_{\mathrm{s}} / B$ ratio of 0.4 the reduction is by $45 \%$.

When a container is exposed to linear shakiness, a series of surface wave trains are formed in the fluid and so the fluid gets displaced, and accordingly applies a time-varying pressure on the peripheral shells of the container. Fig. 15 reveals the pressure distribution curves along the height measured at the middle section of the considered tank model under Kobe earthquake, when the peak base shears due to hydrodynamic forces are gotten. The peak hydrodynamic pressure responses for the cases of the unbaffled tank and the cases of $B / B=0.2$ and 0.4 are, respectively, $0.974,0.815$ and $0.625 \mathrm{kPa}$. In fact, as the baffle width increases, the area below the pressure curve becomes smaller and weaker, leading to a diminished effect of the baffle device on the liquid impulses.

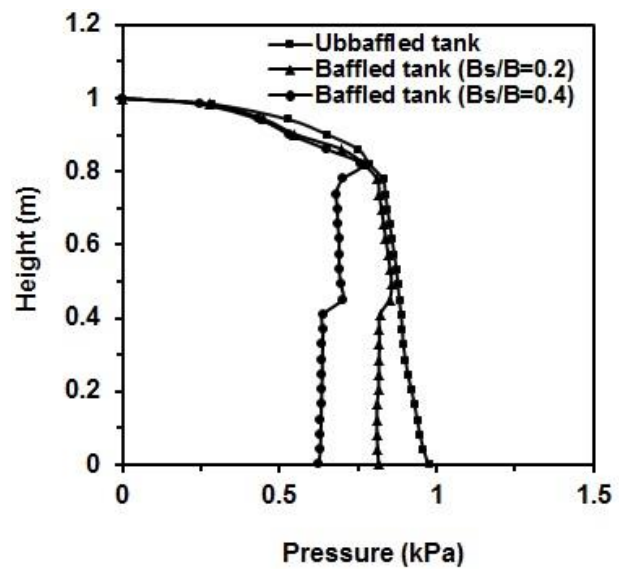

Fig. 15. Variation of hydrodynamic pressure distribution under Kobe record $(h / H=0.4)$.

\section{Conclusions}

In the present paper, a boundary element model has been proposed for the determination of sloshing response in the partially liquid filled rectangular tanks with and without baffles. The BEM was employed along the walls and free surface boundary conditions

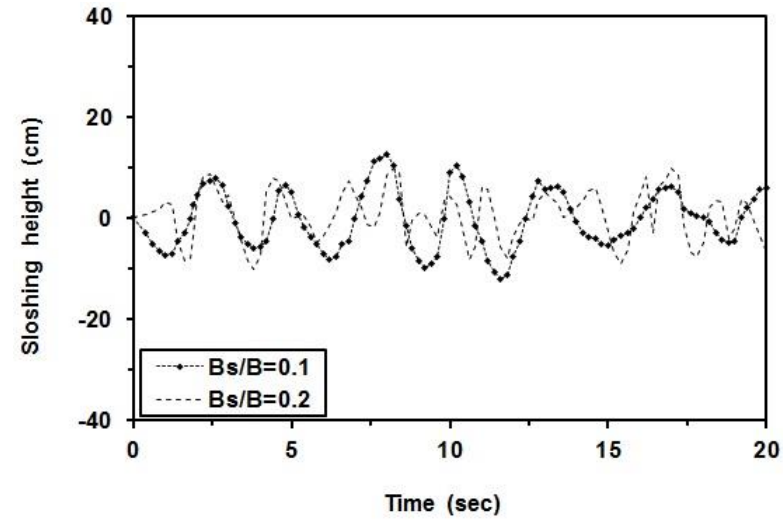

a) $B_{\mathrm{s}} / B=0.1$ and 0.2

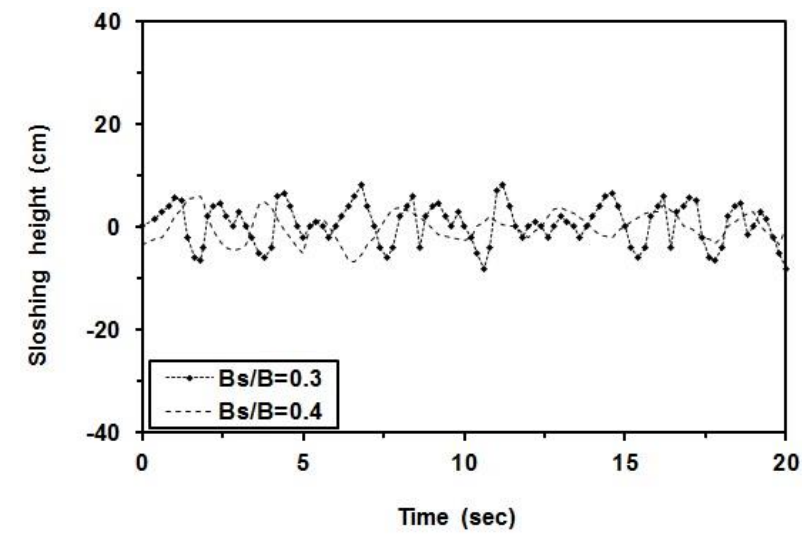

b) $B_{\mathrm{s}} / B=0.3$ and 0.4

Fig. 14. Liquid sloshing time variations for various baffle widths. 
to acquire the equations governing the liquid motion. The proposed numerical scheme acts well in comparison with the analytical solution and published numerical results in the literature. The major improvement of the offered particular form is due to the reduction in the size of computational matrices, which reduces the computational costs. Several parametric studies are performed to assess the performance of baffled liquid tanks subjected to the horizontal and vertical excitations with different excitation frequencies and excitation amplitudes. The following conclusions may be drawn as the results obtained based on the analyses:

1. With the installation of baffle beneath liquid free surface, it is noticed that maximum sloshing heights are significantly reduced which can be because of dissipating the kinetic energy of moving fluid.

2. Liquid surface displacement decreases with increase in height. Moreover, the effectiveness of baffles increases with the increase of their width. This is because of the self-damping property of the liquid by which it absorbs the energy provided at the walls.

3 . The peak sloshing displacement show relatively remarkable increase when the sloshing natural frequency approaches the frequency of the forced excitation.

\section{References}

1- Ibrahim, R.A.; (2005), Liquid Sloshing Dynamics: Theory and Applications; Cambridge University Press, New York.

2. Evans D.V., McIver P. (1987), Resonant frequencies in a container with a vertical baffle, Journal of Fluid Mechanics, 175: p. 295-307.

3. Watson E.B.B., Evans D.V., (1991), Resonant frequencies of a fluid in containers with internal bodies, Journal of Engineering Mathematics, 25: p. 115-135.

4. Choun, Y.-S., Yun, C.-B., (1996), Sloshing characteristics in rectangular tanks with a submerged block, Computers \& Structures, 61(3): p. 401-413.

5. Choun, Y.-S., Yun, C.-B., (1999), Sloshing analysis of rectangular tanks with a submerged structure using small-amplitude wave theory. Earthquake Engineering \& Structural Dynamics, 28(7): p.763-783.

6. Warnitchai, P., Pinkaew T., (1998), Modelling of liquid sloshing in rectangular tanks with flowdamping devices. Engineering Structures, 20: p. 593600.

7. Isaacson, M., Premasiri S., (2001), Hydrodynamic damping due to baffles in a rectangular tank. Canadian Journal of Civil Engineering, 28: p. 608616.

8. Biswal, K.C., Bhattacharyya, S.K., Sinha, P.K., (2003), Free vibration analysis of liquid filled tank with baffles. Journal of Sound and Vibration, 259 (1): p.177-192.
9. Biswal, K.C., Bhattacharyya, S.K., Sinha, P.K., (2004), Dynamic response analysis of a liquid filled cylindrical tank with annular baffle. Journal of Sound and Vibration, 274 (1-2), p. 13-37.

10. Frandsen, J. B., (2004), Sloshing motions in excited tank, Journal of Computational Physics, 196(1), p. 53-87.

11. Bermudez, A., Rodriguez, R., Santamarina, D., (2003), Finite element computation of sloshing modes in containers with elastic baffles plates. International Journal for Numerical Methods in Engineering, 56(3): p. 447-467.

12. Lee, S.Y., Cho, J.R., (2003), Dynamic analysis of baffled fuel storage tanks using the ALE finite element method. Int. J. Numer. Methods Fluids, 41: p. 185208.

13. Iseki, T., Shinkai, A., Nakatake, K., (1989), Boundary element analysis of 3-dimensional sloshing problem by using cubic spline element. Naval Architect (Japan), 166: p. 355-362.

14. Sen, D. A., (1995), cubic-spline boundary integral method for two-dimensional free-surface flow problems. International Journal for Numerical Methods in Engineering, 38(11): p. 1809-1830.

15. Dutta, S., Laha, M.K., (2000), Analysis of the small amplitude sloshing of a liquid in a rigid container of arbitrary shape using a low-order boundary element method. Int J Numer Meth Engng, 47: p. 1633-48.

16. Gedikli, A., Erguven, M.E., (1999), Seismic analysis of a liquid storage tank with a baffle. Int $\mathbf{J}$ Sound Vib 1999, 223: p. 141-56.

17. Gedikli, A., Erguven, M.E., (2003), Evaluation of sloshing problem by variational boundary element method, Engineering Analysis with Boundary Elements, 27(9): p. 935-943.

18. Kita, E., Katsuragawab, J., Kamiya, N., (2004), Application of Trefftz-type boundary element method to simulation of two-dimensional sloshing phenomenon, Engineering Analysis with Boundary Elements, 28(6): p. 677-683.

19. Jamali, M., (2006), BEM modeling of surface water wave motion with laminar boundary layers. Engineering Analysis with Boundary Elements, 30(1): p. 14-21.

20. Ning D.-Z., Teng B., Zhao H.-T., and Hao C.-I., (2010), A comparison of two methods for calculating solid angle coefficients in a BIEM numerical wave tank, Engineering Analysis with Boundary Elements, 34(1): p. 92-96.

21. Sygulski, R., (2011), Boundary element analysis of liquid sloshing in baffled tanks, Engineering Analysis with Boundary Elements, 35 (8): p. 978-983. 22. Ebrahimian, M., Noorian, M.A., Haddadpour, H., (2013), A successive boundary element model for investigation of sloshing frequencies in axisymmetric multi baffled containers, 37(2): p. 383-392. 
23. De-Zhi, N., Wei-Hua, S., Yu-Long, L., Bin, Teng, (2012), Boundary Element Investigation of Liquid Sloshing in Coupled Horizontal and Vertical Excitation, Journal of Applied Mathematics, 20 pages. 24. Firouz-Abadi, R.D., Haddadpour, H., Noorian, M.A., Ghasemi, M., (2008), A 3D BEM model for liquid sloshing in baffled tanks, Int. J. Numer. Methods Eng., 76: p. 1419-1433.

25. Shekari, M.R., Khaji, N., Ahmadi, M.T., (2009), A coupled BE-FE study for evaluation of seismically isolated cylindrical liquid storage tanks considering fluid-structure interaction. Journal of Fluids and Structures, 25(3): p. 567-585.

26. Shekari, M.R., (2014), Seismic response of liquidfilled tank with baffles, Journal of Marine Science and Application, Volume 13: p. 299-304.

27. Brebbia, C.A., Dominguez, J., (1992), Boundary Elements, An Introductory Course. CMP \& McGrawHill, New York.
28. Biswal, K.C., Bhattacharyya, S.K., Sinha, P.K. (2006), Non-linear sloshing in partially liquid filled containers with baffles. Int J Numer Methods Eng, 68: p. 317-337.

29. Goudarzi, M.A., Sabbagh-Yazdi, S.R., (2012), Investigation of nonlinear sloshing effects in seismically excited tanks. Soil Dynamics and Earthquake Engineering, 43, p. 355-365.

30. Degtyarev, K., Gnitko, V., Naumenko, V., Strelnikova, E. A., (2016), Reduced Boundary Element Method for Liquid Sloshing Analysis of Cylindrical and Conical Tanks with Baffles. International Journal of Electronic Engineering and Computer Science, 1: p. 14-27.

31. Akyildiz, H., Unal, E.N., (2005), Experimental investigation of pressure distribution on a rectangular tank due to the liquid sloshing, Ocean Engineering, 32, p. 1503-1516. 